\title{
Studies on the Preparation and in vitro - in vivo Evaluation of Mucoadhesive Microspheres of Glycyrrhetinic Acid Isolated from Liquorice
}

\author{
Sharad Visht and G. T. Kulkarni \\ Department of Pharmaceutical Technology, Meerut Institute of Engineering and Technology, Meerut, \\ Uttar-Pradesh, India
}

Received: November 06, 2014; Accepted: January 05, 2015

\begin{abstract}
The present work deals with the formulation and evaluation of mucoadhesive microspheres containing glycyrrhetinic acid. Mucoadhesive microspheres were prepared by single phase emulsification method and heat stabilization method. Chemical stabilization appeared as fast and simple technique for producing glycyrrhetinic acid loaded microspheres. Microspheres prepared by this method produced sufficient production yield, high drug encapsulation efficiency and reproducibility. Among the different formulations, microspheres with drug - polymer ratio of 1:1 (C1) was found to have the maximum mucoadhesive property as well as in vitro and in vivo drug release property. Therefore, it could be considered as the best formulation.
\end{abstract}

Key words: Mucoadhesive, microspheres, glycyrrhetinic acid, chemical stabilization, polymer, liquorice

\section{Introduction}

The evolution of drug delivery systems has indicated microspheres as an important part of novel drug delivery systems. Microspheres are solid and spherical particles that range in size of $1-1000 \mu \mathrm{m}$. They are made of natural or synthetic polymers. With enormous applicability of microspheres, they are becoming a realistic approach to drug delivery. However, the success of microspheres as a drug delivery system is limited due to their short sustaining time at the site of absorption. This limitation can be overcome by incorporating a mucoadhesive to the microspheres, which would be extending the sustaining time of microspheres at the site of absorption by providing an intimate adhesive contact of the drug delivery system with the absorbing membranes (Shukla and Tiwari, 2012; Sinha et al., 2004; Sivadas et al., 2008).

Mucoadhesive microspheres have efficient absorption and enhanced bioavailability of the drugs due to a high surface to volume ratio, a much more intimate contact with the mucus layer and specific targeting of drugs to the absorption site. The concept of mucoadhesion is most widely utilized in novel drug delivery systems. The mucoadhesion is the adhesive interactions between polymers and mucus or mucosal surfaces. It refers to the state in which two surfaces, at least one biological in nature gets held together in close contact by interfacial forces for an extended duration of time (Chun et al., 2005b; Chun et al., 2005a;, Soane et al., 1999; Sun et al., 2009).

Among the serious health problems of global concern, gastric hyperacidity and ulceration of the stomach mucosa are very common. Peptic ulcer diseases (encompassing gastric ulcer and duodenal ulcer) are induced by several factors, including stress, smoking, nutritional deficiencies, and ingestion of non-steroidal anti-inflammatory drugs. Peptic ulcer results due to imbalance between offensive (acid, pepsin and Helicobacter pylori) and defensive factors (mucin, prostaglandin, bicarbonate, nitric oxide and growth factors) (Hall, 2010). Mostly two main approaches are utilized for treating peptic ulcer. The first deals with reducing the production of gastric acid and the second with supporting gastric mucosal protection (Laurence et al., 2011). Commonly available anti-ulcer drugs such as $\mathrm{H}_{2}$ receptor antagonists, proton pump inhibitors and cytoprotectants have many side effects and therapeutic limitations (Tripathi, 2008; Brenner and 
Stevens, 2009). Therefore, natural substances having antiulcer properties are preferred. Several plants and herbs have been used to treat gastrointestinal disorders, including gastric ulcers. One of the indigenous plants, Glycyrrhiza glabra Linn. (family: Leguminosae) is known to possess components that have antipeptic-ulcer activity (N and Chopra, 2006; Khare, 2007). The roots and rhizomes of G. glabra known as licorice is one of the most extensively researched medicinal plants and has a history of consumption for the past 6000 years (Mukherjee et al., 2010). Glycyrrhetinic acid is a beta-amyrin type triterpenoid compound having several activities like antiulcer, antibacterial, antifungal, antiviral, anticancer etc. Therefore, this paper deals with preparation and evaluation of mucoadhesive microspheres of glycyrrhetinic acid for the treatment of peptic ulcer.

\section{Materials and Methods}

Materials: Crude Liquorice was purchased from Meerut market during the month of October (2011) and authenticated by Dr. Anjula Pandey, Principal Scientist, National Bureau of Plant Genetic Resources (NBPGR), New Delhi. The dried of liquorice (G. glabra) was used for extraction of glycyrrhetinic acid as ammonium salt and properly identified. A pure drug sample of glycyrrhetinic acid (CAS Number 471-53-4) was purchased from Sigma Aldrich, USA as a reference compound. All other reagents and chemicals were of analytical grade and purchased from approved chemical vendors only. These reagents and chemicals include bovine serum albumin (BSA), glutaraldehyde, carbopol, liquid paraffin, acetone, silica gel, chloroform, ammonium hydroxide and hydrochloric acid.

\section{Preparation of microspheres:}

Chemical stabilization method: Mucoadhesive microspheres of glycyrrhetinic acid ammonium were prepared by single phase emulsification method. Bovine serum albumin and carbopol 934P were used and glutaraldehyde was used as chemical cross linker. Prescribed amount $(1500 \mathrm{mg})$ of the drug and polymers were dissolved in water. The mixture was added in a beaker containing liquid paraffin and shear was applied $(100 \mathrm{rpm})$ to prepare primary emulsion at $15^{\circ} \mathrm{C}$. Glutaraldehyde was added drop wise for cross linking of polymer at surface. After 6 hours microspheres were separated by centrifugation. At last after washing with acetone thrice, microspheres were dried under vacuum. In this way four microsphere formulations with drug polymer at the ratio of 1:1, 1:2,1:3 and 1:4 were prepared and named as $\mathrm{C} 1, \mathrm{C} 2, \mathrm{C} 3$ and $\mathrm{C} 4$.

Heat stabilization method: In heat stabilization method, heat (raise in temperature condition) was applied for final rigidification and stabilization of the glycyrrhetinic acid loaded microspheres. Bovine serum albumin and carbopol 934P were used. When heat was applied it denaturized the protein at surface. Prescribed amount of drug and polymers were dissolved in water and the mixture was added in a beaker containing liquid paraffin followed by application of shear (100 rpm) to prepare primary emulsion at $15^{\circ} \mathrm{C}$. Consequently when heat was applied, linear increase in temperature up to $70^{\circ} \mathrm{C}$ causes rigidification at the surface. Afterwards $6 \mathrm{hrs}$ centrifugation was done to separate the microspheres. Finally washing was done with acetone thrice and microspheres were dried under vacuum. Similarly, four microspheres formulations in the drug polymer ratio of $1: 1,1: 2,1: 3$ and $1: 4$ were prepared and named as $\mathrm{H} 1, \mathrm{H} 2$, $\mathrm{H} 3$ and $\mathrm{H} 4$.

\section{Characterization of microspheres}

Particle size analysis, uniformity index and elongation ratio: Prepared microspheres were analyzed by optical microscopy to measure its size and shape. At first size was measured in an optical microscope (LEICA DM IL, Switzerland) with a calibrated ocular micrometer by randomly selection around 50 microspheres. Length, width and size of the microspheres were measured. The average size of the microspheres from each batch was expressed as the mean diameter $(\mu \mathrm{m}) \pm$ standard deviation (SD). The shape of the microspheres was estimated by measuring the elongation ratio (ER) which is the quotient of length to breadth of the microspheres. ER $=1.1<\mathrm{ER}<$ 1.15 , and ER $>1.15$ represent a perfect spherical and nonspherical shape, respectively (Das and Ng, 2010).

In order to determine the Uniformity Index (UI), following formula was applied

$\mathrm{UI}=\mathrm{Dw} / \mathrm{Dn}$

Where, Dw and Dn are weight average diameter and number average diameter, respectively, and are calculated as follows: 
$\mathrm{Dw}=\Sigma \mathrm{NiDi}^{4} / \Sigma \mathrm{NiDi}^{3}, \mathrm{Dn}=\Sigma \mathrm{NiDi} / \Sigma \mathrm{Ni}$

Where, Ni is the number of particles with Di diameter.

Where, UI= Uniformity index (below $1.2=$ monodisperse, above $1.2=$ broad particle size distribution).

Scanning electron microscopy (SEM): Scanning electron photomicrographs of drug-loaded microspheres were taken. A small amount of micro-spheres was spread on aluminium stub. Afterwards, the stub containing the sample was placed in the scanning electron microscopy (SEM) chamber. A scanning electron photomicrograph was taken at the acceleration voltage of $30 \mathrm{KV}$, chamber pressure of $0.6 \mathrm{mmHg}$ (Hardenia et al., 2011).

Drug loading and encapsulation efficiency: Samples from each batch of microspheres were dissolved in a phosphate buffer solution ( $\mathrm{pH}$ 7.4) and the actual drug content was determined by UV spectrophotometer (Model-1601, Shimadzu, Japan). Encapsulation efficiency was calculated from the ratio of actual to theoretical drug content and expressed as a percentage (Yadav and Jain, 2011).

Percentage yield: The yield of production was calculated as the amount of microspheres obtained with respect to the theoretical content of microsphere. The calculation of percentage yield was done by using the following formula:

Yield $(\%)=$ Amount of microspheres obtained /Theoretical content x 100 (Yadav and Jain, 2011).

Swelling index: A known weight $(50 \mathrm{mg})$ of microspheres was placed in a glass vial containing $10 \mathrm{ml}$ of distilled water at $37 \pm 0.50{ }^{\circ} \mathrm{C}$ in incubator with occasional shaking. The microspheres were periodically removed, blotted with filter paper and their changes in weights were measured during the swelling until equilibration was attained. Finally, the weight of the swollen microspheres was recorded after a period of 3 hours, and the swelling ratio (SR) was then calculated from the formula (Shivanand et al., 2010).

Swelling Index $=\mathrm{We}-\mathrm{W}_{\mathrm{O}} / \mathrm{W}_{\mathrm{O}}$

Where, Wo = Initial weight of the dry microspheres,

$\mathrm{We}=$ weight of the swollen microspheres at equilibrium swelling in the media.

The studies were carried out in triplicate.
Mucoadhesion study: The mucoadhesive property of the microspheres was evaluated by in vitro adhesion testing method known as wash off method. Piece of goat stomach mucosa $1 \times 1 \mathrm{~cm}$ was tied on to a glass slide using a thread. Approximately 100 microspheres were spread on to the wet rinsed tissue specimen and the prepared slide was hung on to one of the grooves of a USP tablet disintegrating test apparatus. The disintegrating test apparatus was operated whereby the tissue specimen was given up and down movements regularly in the beaker of the disintegrating apparatus, which contained the gastric fluid ( $\mathrm{pH}$ 1.2). At the end of $30 \mathrm{~min}, 1 \mathrm{hr}$ and thereafter at hourly intervals up to $4 \mathrm{hrs}$, the number of microspheres still adhering to the tissue was counted (Hardenia et al., 2011).

Percent mucoadhesion $=($ weight of adhered microspheres/weight of applied microspheres $) \times 100$.

In vitro drug release: The drug release study was carried out using USP paddle type apparatus at $37 \pm 0.5^{\circ} \mathrm{C}$ with $100 \mathrm{RPM}$ using $900 \mathrm{ml}$ of $0.1 \mathrm{~N} \mathrm{HCL}$ solution as a dissolution medium. Five millilitre of aliquot was withdrawn at a pre-determined time intervals, up to $12 \mathrm{hrs}$. The medium was replaced with $5 \mathrm{ml}$ of fresh buffer each time. The absorbance was measured by UV spectrophotometer at the wavelength of $291 \mathrm{~nm}$ and \% cumulative release of the formulations was calculated (Shivanand et al., 2010).

In vivo studies: In vitro studies and evaluation of the investigated microspheres were further approved by in vivo study. In vivo study was conducted in accordance to the guidelines prescribed by Committee for the Purpose of Control and Supervision of Experiments on Animals (CPSCEA), India. Male albino rats of both sexes were selected and divided with three groups comprising minimum of 3 rats. All the animals were housed in standard cages. Group A was considered as Control group (positive control) administered with $80 \%$ ethanol. Group $\mathrm{B}$ and Group C were administered with pure femotidine (standard) and mucoadhesive microspheres $\mathrm{C}$, respectively. Peptic ulcer was induced by administering (80\% ethanol) as necrotizing agent as well established for producing gastric lesions. All animals were administered by gastric intubation method. Assuming the gastric emptying time of fasted rats as 30 minutes pure glycrytinic acid and formulation $\mathrm{C} 1$ was administered 30 minutes 
before the administration of the necrotizing agent. Rats were sacrificed thereafter under ether anesthesia $1 \mathrm{hr}$ after treatment with ulcerogenic agent or necrotizing agent. Stomachs of the rats were excised and opened along the greater curvature. Excised stomach parts were washed with normal saline and lesions were quantified using a dissecting microscope with square grid eye piece to assess the formation of ulcers. Ulcerated as well as total areas were measured as $\mathrm{mm}^{2}$ for each stomach and ulcer index were calculated using the following formula:

Ulcer Index $=$ [Ulcerated area/Total stomach area] X 100.

\section{Results and Discussion}

Preparation, drug loading and encapsulation efficiency of microspheres: Glycyrrhetinic acid loaded microspheres were produced with high drug encapsulation efficiency. Chemical stabilization technique was generally characterized by high drug encapsulation efficiency. The yield of the production obtained in the range from 95 to 99 $\%$ (Table 2) for both chemical stabilization and heat stabilization techniques.

C 1

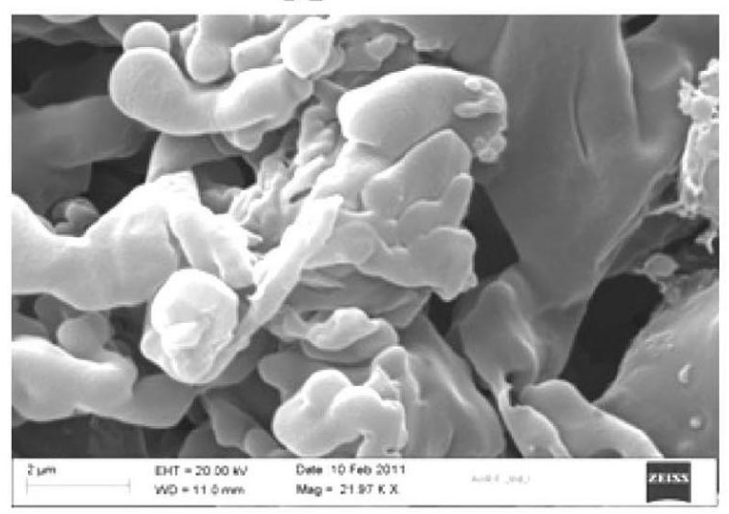

C 3

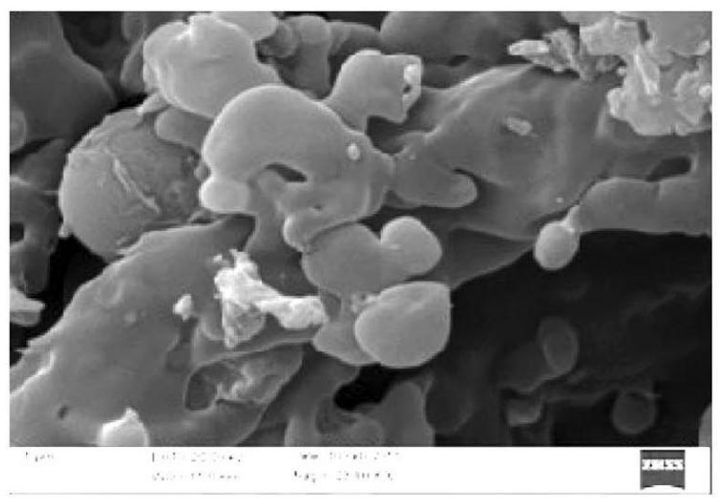

\section{Characterization of microspheres}

Particle size analysis \& Scanning Electron Microscopy (SEM): The particle size of each microsphere formulation coded ( $\mathrm{C} 1$ to $\mathrm{C} 4 \& \mathrm{H} 1$ to $\mathrm{H} 4)$ are reported in Table 1. Particle sizes of the microsphere formulations ranged from 2.87 to $7.23 \mathrm{~mm}$. All the microspheres were found to be spherical to non-spherical and none were found to be perfect spherical. SEM micrographs of the microsphere formulations coded $\mathrm{C} 1$ to $\mathrm{C} 4$ reported in Figure 1. The microspheres exhibited irregular shape and crumpled surface. They seemed to be hollow microspheres, which collapsed during the preparation process. The micrographs belonging to the formulations $\mathrm{H} 1$ and $\mathrm{H} 4$ are shown in Figure 2. These microspheres exhibited spherical shape and smooth surface. The results showed that the drug/polymer ratio affected the morphological characteristics of the spray dried microspheres extensively. As the polymer ratio increased, more spherical microspheres with smoother surface were obtained.

Figure 1. SEM Photograph of formulations C1, C2, C3 and C4. 
H 1

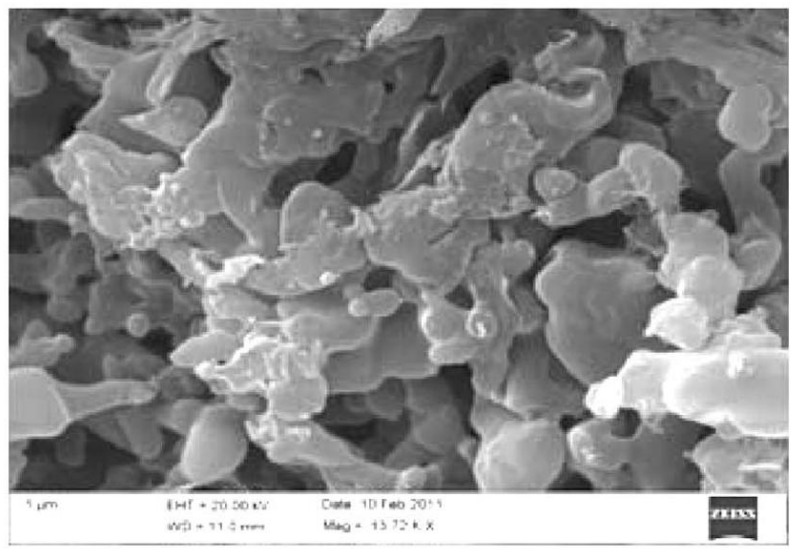

H 3

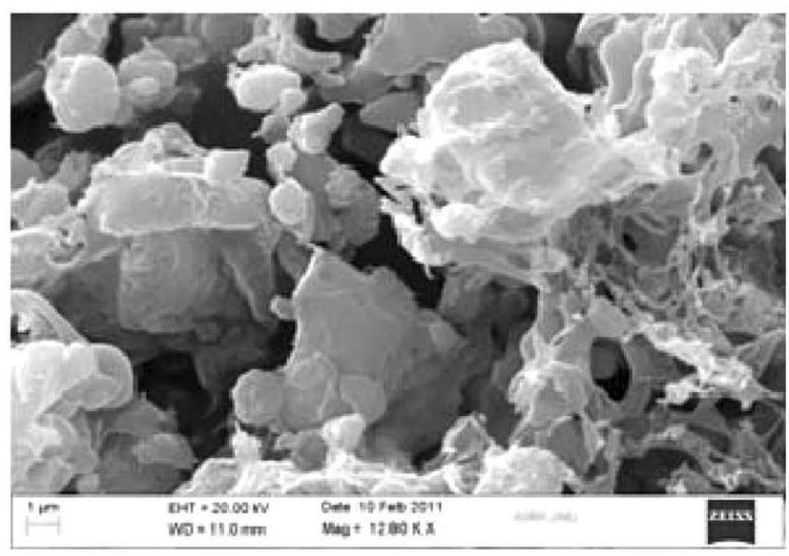

H 2

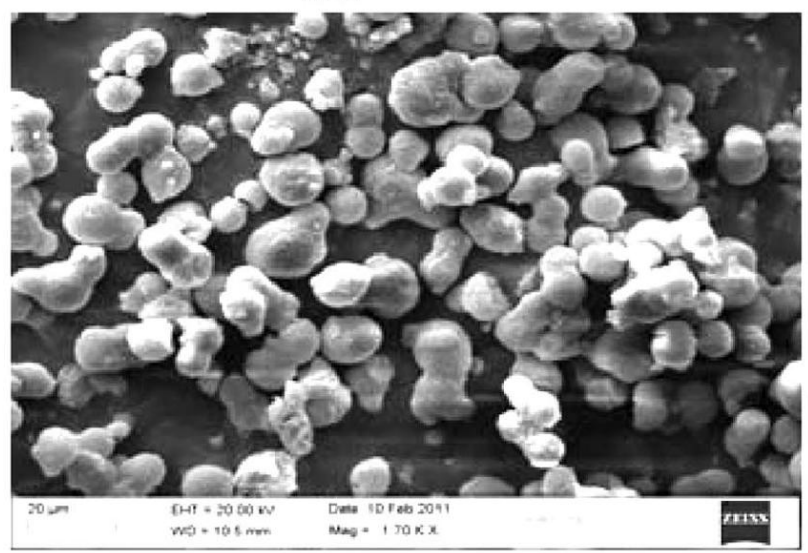

H 4

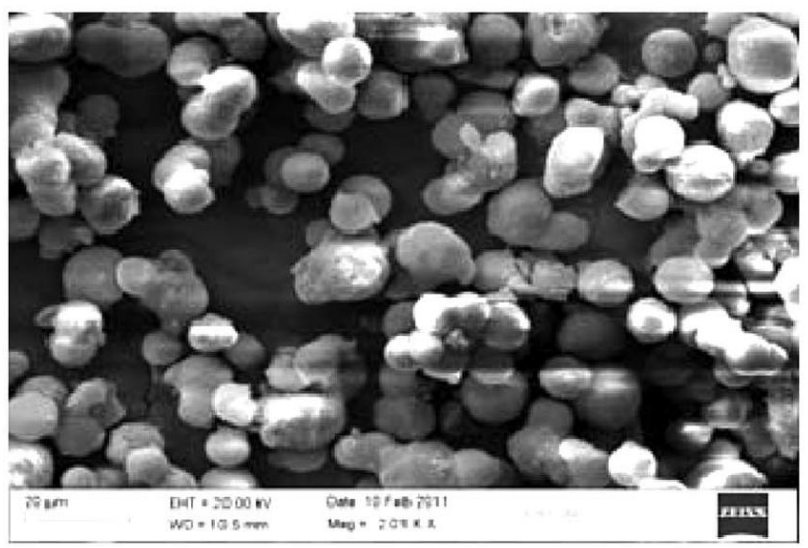

Figure 2. SEM Photograph of formulations H1, H2, H3 and H4.

Percentage yield and swelling index: Percentage yield and swelling index of the microspheres are shown in table 1. The results clearly showed that the all the formulations had sufficient swelling property as well percentage yield. Formulation $\mathrm{C} 1$ and $\mathrm{C} 2$ had the highest swelling and percentage yield, respectively.

Mucoadhesive property and in vitro drug release: Percentages of mucoadhesion shown by microspheres are given in Table 2. The results showed that the microspheres had good mucoadhesive properties and could adequately adhere on stomach mucosa. The results also showed that the microspheres prepared by chemical stabilization method had better mucoadhesive property than those prepared by heat stabilization method. Since the highest percentage $(76 \pm 1.92)$ was obtained with $\mathrm{C} 1$ formulation, it was considered to be with good mucoadhesive property.

In vitro release profiles of glycyrrhetinic microsphere formulations are shown in Figure 3 and Table 3. The percentage of the drug released was not same for all formulations. The formulations coded $\mathrm{C} 1$ (drug/polymer ratio $1: 1$ ) and $\mathrm{C} 4$ (drug/polymer ratio 1:4) were found to be the highest and the lowest release profiles, respectively. This result showed that drug/polymer ratio affected the release rate of the drug. As the polymer amount increases, the release rates of drugs decreases.

In vivo study: The microsphere $\mathrm{C} 1$ was found to be good protection against gastric ulcers as observed in vivo studies. Mucoadhesive properties as well as well protective property of glycrytinic acid made this formulation effective against peptic ulcer. Its histopathology Figure 4 and ulcerative index shows that formulation $\mathrm{C} 1$ shows significant decrease in ulcer index $(\mathrm{P}<0.001)$ as compared to standard group. Presence of ample of mucin and hence favoring the mucoadhesive property of the investigated microspheres $\mathrm{C} 1$ makes it a successful delivery system for treating peptic ulcer. 


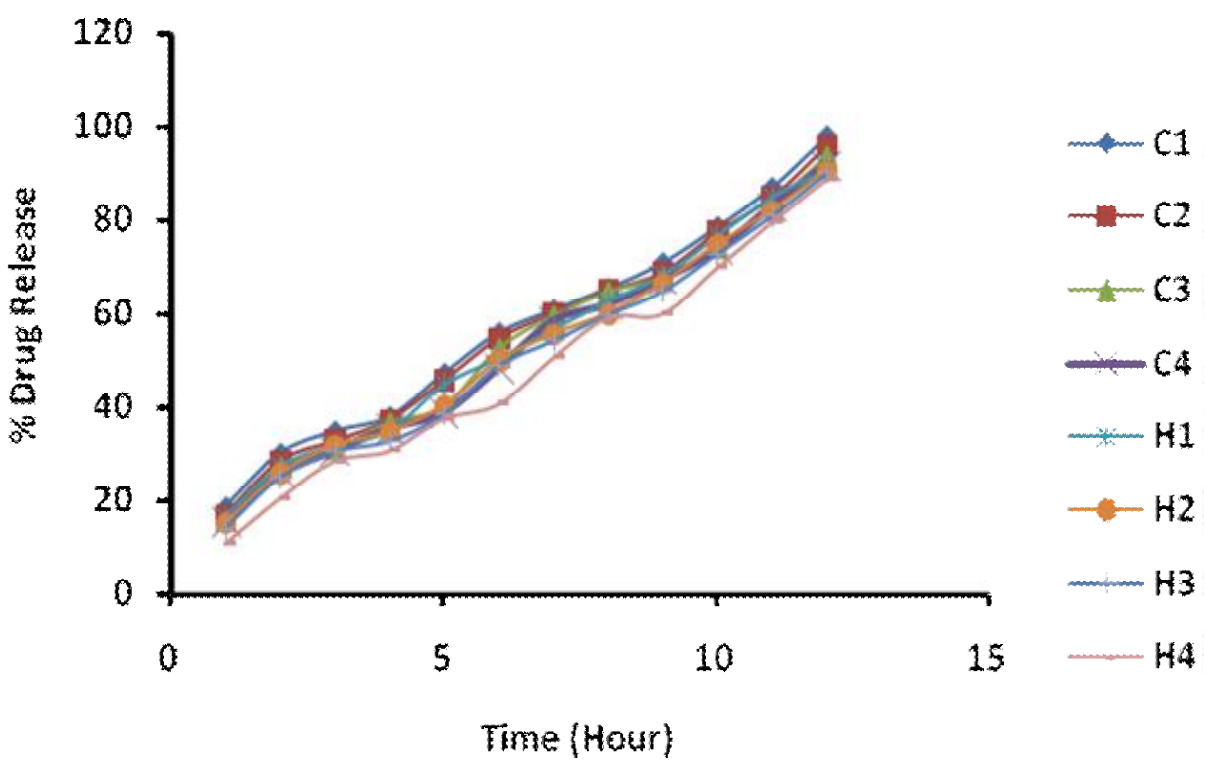

Figure 3. In vitro drug release at $\mathrm{pH} 1.2$.

Table 1. Particle size, uniformity index, elongation ratio and shape of microspheres.

\begin{tabular}{|c|c|c|c|c|}
\hline Formulation & $\begin{array}{l}\text { Particle size }(\mu \mathrm{m}) \\
\pm \mathrm{SD}\end{array}$ & UI & ER & Shape \\
\hline $\mathrm{C} 1$ & $6.32 \pm 0.12$ & 1.321 & $1.23 \pm 0.06$ & Non spherical \\
\hline $\mathrm{C} 2$ & $5.39 \pm 0.17$ & 1.0989 & $1.08 \pm 0.09$ & Spherical \\
\hline $\mathrm{C} 3$ & $4.38 \pm 0.12$ & 1.3097 & $1.18 \pm 0.11$ & Non spherical \\
\hline $\mathrm{C} 4$ & $6.21 \pm 0.19$ & 0.89 & $1.11 \pm 0.09$ & Spherical \\
\hline H1 & $2.87 \pm 0.09$ & 1.898 & $1.21 \pm 0.08$ & Non spherical \\
\hline $\mathrm{H} 2$ & $6.04 \pm 0.12$ & 1.005 & $1.09 \pm 0.04$ & Spherical \\
\hline H3 & $3.89 \pm 0.09$ & 0.99 & $1.11 \pm 0.10$ & Spherical \\
\hline $\mathrm{H} 4$ & $7.23 \pm 0.08$ & 0.9933 & $1.06 \pm 0.09$ & Spherical \\
\hline
\end{tabular}

Where UI= Uniformity index (below $1.2=$ monodisperse, above $1.2=$ broad particle size distribution), ER= Elongation ratio $(\mathrm{ER}=1$, Perfect Spherical $/ 1<\mathrm{ER}<1.5=$ Sperical $/ \mathrm{ER}>1.15=$ non spherical

Table 2. Percentage yield, encapsulation efficiency, loading capacity, swelling index and \% mucoadhesion.

\begin{tabular}{cccccc}
\hline Formulation & Percentage yield & EE & LC & Swelling index & $\begin{array}{c}\text { Mucoadhesion after } \\
10 \mathrm{hr}\end{array}$ \\
\hline C1 & $98.33 \pm 0.84$ & 98.33 & 50.12 & $0.98 \pm 0.13$ & $76 \pm 1.92$ \\
C2 & $99.0 \pm 1.12$ & 99.0 & 50.34 & $0.23 \pm 0.02$ & $64 \pm 1.23$ \\
C3 & $96 \pm 0.74$ & 96.0 & 33.34 & $0.97 \pm 0.12$ & $70 \pm 1.42$ \\
C4 & $96.33 \pm 1.08$ & 96.33 & 34.78 & $0.19 \pm 0.1$ & $56 \pm 1.4$ \\
H1 & $97.33 \pm 0.82$ & 97.33 & 52.34 & $0.98 \pm 0.2$ & $67 \pm 2.1$ \\
H2 & $97.66 \pm 0.94$ & 97.66 & 49.56 & $0.23 \pm 0.2$ & $54 \pm 1.43$ \\
H3 & $95.57 \pm 1.05$ & 95.57 & 26.66 & $0.97 \pm 0.1$ & $60 \pm 1.28$ \\
H4 & $95.67 \pm 0.89$ & 95.68 & 32.67 & $0.19 \pm 0.11$ & $49 \pm 13$ \\
\hline
\end{tabular}

Where, $\mathrm{EE}=$ Encapsulation efficiency, $\mathrm{LC}=$ Loading capacity 
Table 3. In vitro drug release at $\mathrm{pH} 1.2$.

\begin{tabular}{ccccccccccccc}
\hline Formulation & $1 \mathrm{hr}$ & $2 \mathrm{hr}$ & $3 \mathrm{hr}$ & $4 \mathrm{hr}$ & $5 \mathrm{hr}$ & $6 \mathrm{hr}$ & $7 \mathrm{hr}$ & $8 \mathrm{hr}$ & $9 \mathrm{hr}$ & $10 \mathrm{hr}$ & $11 \mathrm{hr}$ & $12 \mathrm{hr}$ \\
\hline C1 & 18.57 & 30.14 & 34.89 & 37.99 & 47.31 & 55.98 & 60.87 & 65.45 & 70.89 & 78.56 & 87 & 98 \\
C2 & 16.99 & 28.56 & 32.88 & 37.41 & 45.89 & 54.86 & 60.21 & 65.01 & 68.99 & 77.55 & 85 & 96 \\
C3 & 15.78 & 27.41 & 31.11 & 36.88 & 40.29 & 52.76 & 59.76 & 64.89 & 68.01 & 74.34 & 83.46 & 94 \\
C4 & 15.41 & 25.66 & 30.77 & 35.56 & 38.99 & 48.54 & 58.57 & 62.11 & 67.45 & 73.69 & 83.01 & 92 \\
H1 & 16.76 & 27.44 & 32.11 & 35.11 & 45.11 & 50.65 & 56.98 & 62.90 & 68.31 & 76.77 & 85.11 & 91 \\
H2 & 15.42 & 25.89 & 31.99 & 34.89 & 40.56 & 50.12 & 55.76 & 60.18 & 66.56 & 74.89 & 82.11 & 90.67 \\
H3 & 14.11 & 24.98 & 30.56 & 32.79 & 38.57 & 48.99 & 54.31 & 59.87 & 64.89 & 72.90 & 80.90 & 90.10 \\
H4 & 10.99 & 20.61 & 28.67 & 30.88 & 37.78 & 40.89 & 50.87 & 59.45 & 60.23 & 70.11 & 79.77 & 89 \\
\hline
\end{tabular}
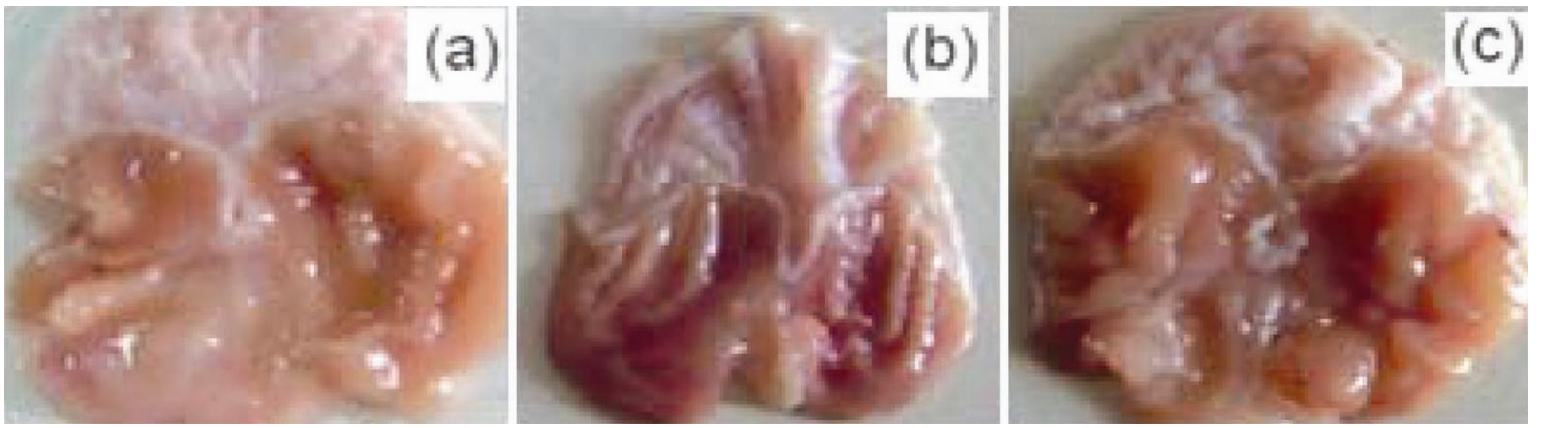

Figure 4. In vivo studies demonstrating effectiveness of $\mathrm{C} 1$.

\section{Conclusion}

Chemical stabilization was found to be a fast and simple technique for producing glycyrrhetinic acid loaded microspheres where they produced sufficient yield, high drug encapsulation efficiency and reproducibility from batch to batch. Owing to the observed high encapsulation efficiency and sufficient production yield, it can be concluded that chemical stabilization method was a simple and suitable technique for producing glycyrrhetinic acid loaded microspheres. All the microspheres were at a suitable size and had good mucoadhesive property for administration.

It was concluded from the studies that the microspheres prepared by chemical stabilization and having drug:polymer ratio of 1:1 (C1) was best with respect to the in vitro drug release and mucoadhesive properties. The microspheres with drug:polymer ratio of 1:1 had the maximum mucoadhesive property as well as the drug release property, therefore, it was considered as the best formulation. Further in vivo studies also confirmed its potential property evaluated by in vitro studies. Thus in vivo study also revealed the mucoadhesive microspheres as effective drug delivery system for treating peptic ulcer.

\section{Declaration of interest}

The authors declare that there is no any conflict of interest.

\section{References}

Brenner, G.M. and Stevens, C. 2009. Pharmacology, Elsevier Health Sciences.

Chun, M.K., Cho, C.S. and Choi, H.K. 2005a. Mucoadhesive microspheres prepared by interpolymer complexation and solvent diffusion method. Int. J. Pharm. 288, 295-303.

Chun, M.K., Sah, H. and Choi, H.K. 2005b. Preparation of mucoadhesive microspheres containing antimicrobial agents for eradication of H. pylori. Int. J. Pharm. 297, 172-179.

Das, S. and Ng, K.Y. 2010. Colon-specific delivery of resveratrol: Optimization of multi-particulate calciumpectinate carrier. Int. J. Pharm. 385, 20-28.

Laurence L.B., Bruce A.C., Björn C.K. 2011. Goodman and Gilman's The Pharmacological Basis of Therapeutics, McGraw-Hill Medical.

Hall, J.E. 2010. Guyton and Hall Textbook of Medical Physiology: Enhanced E-book, Elsevier Health Sciences. 
Hardenia, S.S., Jain, A., Patel, R. and Kaushal, A. 2011. Formulation and Evaluation of Mucoadhesive Microspheres of Ciprofloxacin. J. Adv. Pharm. Edu. Res. 1, 214-224.

Khare, C.P. 2007. Indian Medicinal Plants: An Illustrated Dictionary, Springer.

Mukherjee, M., Bhaskaran, N., Srinath, R., Shivaprasad, H.N., Allan, J. J., Shekhar, D. and Agarwal, A. 2010. Anti-ulcer and antioxidant activity of GutGard ${ }^{\mathrm{TM}}$. Indian J. Exp. Biol. 48, 269-274.

N, C. R. and Chopra, I. C. 2006. Indigenous Drugs of India, Academic Publishers.

Shivanand, P., Viral, D., Manish, G., Muzaffar, F. and Venkateswaramurthy, N. 2010. Formulation and Evaluation of Mucoadhesive Microspheres of Amoxicillin Trihydrate by using Eudragit RS 100. Int. J. Chem. Tech. Res. 2, 466470.

Shukla, R.K. and Tiwari, A. 2012. Carbohydrate polymers: Applications and recent advances in delivering drugs to the colon. Carbohydrate Polymers, 88, 399-416.

Sinha, V.R., Singla, A.K., Wadhawan, S., Kaushik, R., Kumria, R., Bansal, K. and Dhawan, S. 2004. Chitosan microspheres as a potential carrier for drugs. Int. J. Phar. 274, 1-33.
Sivadas, N., O'rourke, D., Tobin, A., Buckley, V., Ramtoola, Z., Kelly, J.G., Hickey, A.J. and Cryan, S.-A. 2008. A comparative study of a range of polymeric microspheres as potential carriers for the inhalation of proteins. Int. J. Pharm. 358, 159-167.

Soane, R.J., Frier, M., Perkins, A.C., Jones, N.S., Davis, S.S. and Illum, L. 1999. Evaluation of the clearance characteristics of bioadhesive systems in humans. Int. J. Pharm. 178, 5565 .

Sun, L., Zhou, S., Wang, W., Li, X., Wang, J. and Weng, J. 2009. Preparation and characterization of porous biodegradable microspheres used for controlled protein delivery. Colloids and Surfaces A: Physicochemical and Engineering Aspects, 345, 173-181.

Tripathi, K.D. 2008. Essentials of Medical Pharmacology, Jaypee Brothers Medical Publishers.

Yadav, A. and Jain, D.K. 2011. Formulation and evaluation of mucoadhesive microspheres of propranolol hydrochloride for sustained drug delivery. Asian J. Pharm. .Med. Sci, 1, $1-8$. 